\title{
Une seule éthique ne suffit pas
}

\section{Jean Martin}

Dr med., membre de la rédaction

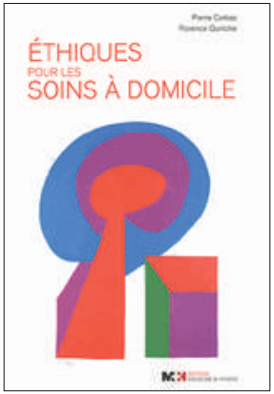

Pierre Corbaz et Florence Quinche Ethiques pour les soins à domicile

Genève: Editions Médecine et Hygiène; 2015.

269 pages. $28 \mathrm{CHF}$. ISBN 978-2-880-49383-7

jean.martin[at]saez.ch
Pierre Corbaz est médecin généraliste à Lausanne et a présidé la Fondation lausannoise des soins à domicile ainsi que sa commission d'éthique, dont l'ouvrage discuté ici donne un reflet des travaux. Il s'est aussi acquis un doctorat en philosophie et a écrit plusieurs livres traitant d'éthique médicale, notamment en fin de vie. Florence Quinche est philosophe et enseigne à la Haute Ecole Pédagogique de Lausanne; elle a été membre de plusieurs commissions d'éthique. Ces deux auteurs collaborent de longue date et apportent aujourd'hui une somme substantielle présentant leur expérience, avec d'autres, dans le conseil éthique au sein de structures de soins à domicile. "Ce livre traite d'un choix de sujets qui se sont illustrés dans les travaux de la commission d'éthique [de la Fondation Soins Lausanne] par leur fréquence, leur acuité, leur originalité ou leur caractère exemplaire. Nous avons estimé qu'il était utile de travailler ces questions et de les mettre à la disposition de collègues». L'ouvrage de près de 300 pages denses est structuré en 12 chapitres. Chacun commence par une ou plusieurs vignettes cliniques, discutées ensuite dans leurs divers aspects au fil des sections du chapitre. Quand le sujet s'y prête, sont incluses les dispositions légales pertinentes, cantonales vaudoises et/ou fédérales suisses.

Il importe de noter que, dans le titre, Ethiques est au pluriel. Les auteurs à ce propos: «Nous sommes tentés d'être en quête de recettes. C'est un leurre parce que l'éthique qui nous intéresse entre en action lorsque justement les recettes s'épuisent. Une autre raison réside dans le fait qu'il n'y a pas qu'une éthique mais plusieurs; plusieurs grammaires éthiques pourrait-on dire.» «Un regard éthique accepte ainsi de se laisser déranger et de sortir des automatismes de la pensée, que ces derniers soient du domaine du soin ou se rattachent à une école philosophique.»

On peut saluer cette position, rappelant celle de l'universitaire genevois Eric Fuchs pour qui l'éthique, c'est répondre à la question "Comment faire pour bien faire?». On ne saurait faire dans la réalité pratique clinique avec un seul «set» fixe et fini de règles. Il est parfois temps, disent Corbaz et Quinche, «de quitter les aspects rigoureux des lois et des règles pour ouvrir la réflexion au doute, au pourquoi, au perpétuel questionnement individualisé.»

A propos de evidence based nursing/medicine: «La pratique basée sur les preuves, ça marche jusqu'au jour où ça ne marche plus, où il n'y pas de bonne solution, où le soignant est mal à l'aise quoi qu'il fasse. La question posée, de plus, ne peut être supprimée, il n'est pas question de s'en débarrasser.» NB: surtout ne pas voir dans ces formulations un côté désabusé. Au contraire: c'est là que sont les défis, auxquels il est d'abord nécessaire mais aussi stimulant, engageant, de devoir répondre, dans un certain malaise voire une souffrance confronté qu'on est souvent à des doubles contraintes et autres dilemmes. Cette position des auteurs doit plutôt donner envie de se plonger dans le livre!

\section{Ethiques pour les soins à domicile n'est pas} le livre que vous lirez en une fois, d'un bout à l'autre.

Parmi les thèmes abordés: justice et soins à domicile, soins et vie privée, secret professionnel, autonomie, discernement et curatelle, l'éthique du «care» et de la sollicitude, maltraitance (des patients, mais aussi parfois des soignants), soins palliatifs à domicile, les problématiques du suicide assisté et de l'assistance sexuelle-sur lesquelles les auteurs ne cachent pas leur perplexité.

Ethiques pour les soins à domicile n'est pas le livre que vous lirez en une fois, d'un bout à l'autre. C'est plutôt un manuel, une somme de référence à laquelle s'adresser quand les situations rencontrées n'admettent pas d'issue aisée, interpellent par leur complexité. On lira alors avec profit les sections du livre correspondant aux domaines, articulations, enjeux correspondants. Dans sa conclusion: «Soutenir les soignants à domicile n'est donc pas seulement une question de déontologie ou d'éthique médicale, mais une question sociétale qui nous concerne tous.» Au moment où le "tsunami gris» est devenu, dans nos pays, l'enjeu majeur de santé publique pour les décennies à venir et où la prise en charge à domicile est une partie importante des stratégies à mettre en œuvre, on ne saurait mieux dire. 